\title{
Development of Low-Cost Thermal-Fluid Undergraduate Laboratory Exercises Complying with the Washington Accord's Graduate Attributes
}

\author{
AzamCheIdris, MohdRashdanSaad, SuriyadiSojipto, MohdRosdzimin Abdul Rahman
}

\begin{abstract}
Signatories of the Washington Accord has to comply with the accord's definition of a competent engineering graduate. In Malaysia, the Engineering Accreditation Council (EAC) is empowered to oversee all the engineering degree courses offered by all universities to make sure they conform with the Washington Accord. Our university has decided to revamp some of the laboratory exercises in order to comply with EAC's requirements. This article reports the thought process of designing new thermal-fluid laboratory exercises that can provide advanced engineering knowledge using investigative scientific process and complex problem analysis. The cost of the laboratory setup was kept at minimum using open-source software that can visualize and measure the density gradient of the flow-field captured by a simple DSLR camera. The students were able to observe complex flow phenomena that stimulated their interest to read further on related engineering research articles. The students achieved well above average on the course and program outcomes that were set for this course.
\end{abstract}

Index Terms: Washington Accord, Thermal-Fluid, Schlieren.

\section{INTRODUCTION}

Outcome-based education (OBE) has been embedded in engineering education in Malaysia since 2000s in line with the government effort to ensure employability of graduates. The OBE in engineering courses in Malaysia are assessed and accredited by Engineering Accreditation Council (EAC), which is a signatory to the Washington Accord (WA). Malaysia is a full signatory of the WA since 2009 and has been a provisional signatory much earlier than that. In 2012 EAC has published a new manual for accreditation of engineering programme to achieve the twelve WA's graduates attributes [1], [2]. In 2017, EAC updated the manual with small changes while at the same time further emphasizing the twelve attributes [3]. In the EAC's manual, the term graduate attributes are called engineering programme outcomes (PO).

All of the programme outcomes will hopefully contribute to the satisfying career development of the engineer throughout their life [4]. Employability of engineers in Malaysia from the employers perspective have been surveyed by [5] and they found that strong preferences are given to the traits of creative problem solving skill, team work

Revised Manuscript Received on September 14, 2019.

Azam Che Idris, Faculty of Engineering, UPNM, Kuala Lumpur, Malaysia.

Mohd Rashdan Saad, Faculty of Engineering, UPNM, Kuala Lumpur, Malaysia.

Suriyadi Sojipto, Faculty of Engineering, UPNM, Kuala Lumpur, Malaysia.

Mohd Rosdzimin Abd Rahman, Faculty of Engineering, UPNM, Kuala Lumpur, Malaysia. cooperation, smart information management and having entrepreneurial spirit. Thus it is imperative to strictly follow the WA guidelines in re-designing the engineering syllabus in order to reduce the high number unemployment of science, engineering and technical field graduates in Malaysia [6].

A working committee has been formed by the Department of Mechanical Engineering in UPNM in order to review the compliance of every undergraduate laboratory exercise. The working committee has identified a few laboratory topics that needed urgent changes. Among the most frequent comments were that the lab works are not investigative in nature and do not emphasize discipline in independent life-long learning. The students were taught using "recipe" and they do not understand why the methodology of the lab work was arranged as such. Only the development of new thermal-fluid laboratory exercises will be reported in this paper.

The biggest constraint for thermal-fluid laboratory exercises is the cost of the equipment. Thermal-fluid in the laboratory settings concern itself with the measurement and control of temperature, pressure, flow velocity and mass flow rate [7]. Most of scientific grade flow diagnostics apparatuses are expensive. The cheaper ones tend to be quite basic in their capabilities and would not allow for more investigative aspect of the laboratory works. Investigation must be included as an objective in the lab module in order to comply with the EAC requirements.

The high cost of flow diagnostics apparatus is exemplified by a recent quotation for a 16-channel pressure scanner at around RM 80,000. Another quotation was given for thermal infrared camera, which cost around RM 20,000 for basic specification and more than RM 60,000 for scientific research grade specification.

Pressure or temperature sensitive paint (PSP \& TSP) can be a good alternative for measuring the entire flow field but again, they still have quite prohibitive cost. In [8] reported in his thesis that PtTFP powder which is the key ingredient in the paint cost as much as USD 700 per gram. A cheaper alternative, $\mathrm{Ru}(\mathrm{II})$, still cost a whopping USD 140 per gram. This does not include the scientific grade Charge-Coupled Device (CCD) camera that can cost upward of USD 5000 even for a basic 1.4-megapixel camera. This camera is needed to calculate the intensity of photon emission from the paint from which the pressure or temperature measurements are inferred. High cost would also be incurred if Particle Image Velocimetry (PIV) system is used to measure flow velocity

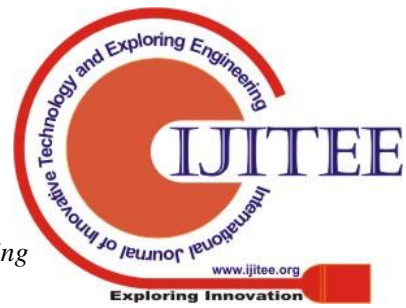




\section{DEVELOPMENT OF LOW-COST THERMAL-FLUID UNDERGRADUATE LABORATORY EXERCISES COMPLYING WITH THE WASHINGTON ACCORD'S GRADUATE ATTRIBUTES}

[9], [10]. A high-resolution camera with very limited speed at $1000 f$ ps can demand price of RM 6000 and above. That has not even include the cost of laser together with synchronizer to time the laser spark with camera shutter. Increasing the image capture frequency will increase the total PIV system cost exponentially.

The expensive nature of thermal-fluid measurement system tends to leave the more modern system specifically for postgraduate research purposes. Thus undergraduate lab would often settled with simple bench or tabletop equipment that can demonstrate only basic thermal-fluid phenomena. This is quite contrary to the aspirations of EAC in its $\mathrm{PO}(\mathrm{i})$ Engineering Knowledge and PO(iv) Investigation. Thus "complex engineering problem" that is related to the forefront professional engineering discipline cannot be discussed effectively at undergraduate level. Undergraduate thermalfluid lab usually settled with cheaper but old apparatus, thus opposing the attributes of $\mathrm{PO}(\mathrm{v})$ Modern Tool Usage [3].

Recently, there has been strong trends to utilize modern computing technology in order to lower the cost of laboratory teaching for undergraduate courses [11]-[16]. Many researchlevel software are released as open-source on the internet, thus allowing highly stimulating laboratory demonstration in the undergraduate level if the software are properly adopted by educators [15], [17], [18]. Using research-level software will allow students to better understand the theory covered in lectures [12].

\section{ADVANCED FLOW DIAGNOSTICS LABORATORY}

\section{A. Planning the Learning Objectives and Outcomes}

The development of new laboratory exercises under thermal-fluid theme was laid out with these aims:

a. Affordable Cost

b. Minimum Setup

c. Field Measurement Capability

d. Complying with $\mathrm{PO}(\mathrm{i}), \mathrm{PO}(\mathrm{ii}), \mathrm{PO}(\mathrm{iv})$ and $\mathrm{PO}(\mathrm{x})$

Besides affordable cost and minimum setup, field measurement was considered important due to the nature of thermal-fluid phenomena. Most phenomena is invisible to the naked eye and a field measurement would give an indication of how the flow interacts with the surroundings besides producing accurate measurement. Vortex formations, turbulence shedding, heat convection, shock waves formation and many other phenomena can be observed clearly by using field measurement. As Ernst Mach once said, "Sehenheißt verstehen" which means "Seeing is Understanding" [19]; thermal-fluid could only be understood properly by directly observing it using flow field contour plot of pressure, temperature, density or velocity.

The learning outcomes (CO) of this lab exercises and its relation to the EAC's programme outcomes (PO) are shown in the Fig. 1. Mainly, there are four COthat strongly contributed to four PO. The ability to understand the theory $\mathrm{CO} 1$, is related to the engineering knowledge PO(i), but at to certain extent also related to attributes of lifelong learning $\mathrm{PO}$ (xii) since the students are required to review related scientific or engineering literatures to understand the experimental works they are required to do. The investigative nature of the experimental works as required by $\mathrm{PO}$ (iv) is satisfied by $\mathrm{CO} 2$ where the students are required to design their own experimental procedures. This is also loosely associated with $\mathrm{PO}(\mathrm{v})$ of using modern tool in learning.

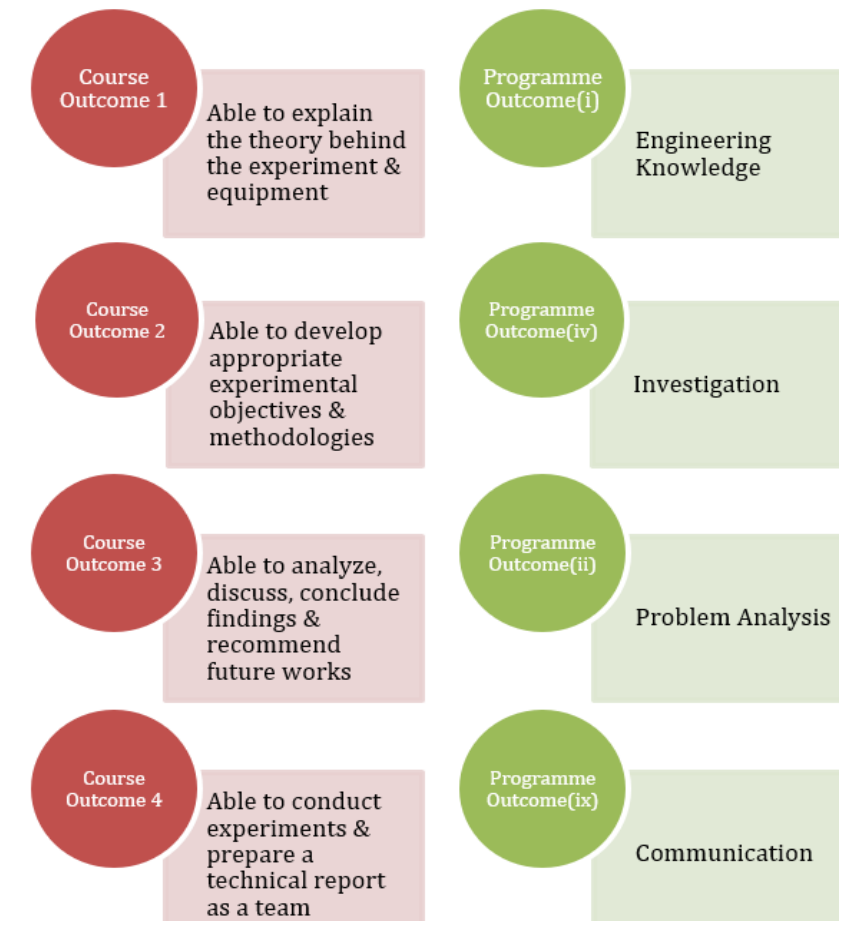

Fig. 1: Relationship between planned Course Outcome (CO) and corresponding Programme Outcome (PO) to be achieved within this course

\section{B. Developing the Laboratory Setup}

The committee decided to develop a background oriented schlieren (BOS) setup that can be used to study different phenomena. BOS was chosen due to their field measurement potential, good flow visualization capability and requires only a camera and suitable background. The basic idea behind BOS is that density changes in some part of the overall flowfield will lead to background image distortion if the camera is focused on the background plane whose normal is perpendicular to the flow direction. The movement of pattern on the background due to changes in density can be calculated using cross-correlation software typically used in the PIV system (see Fig. 2). Typically, the user will define the region of interest (ROI) inside a series of images captured between constant time gap and the crosscorrelation algorithm will detect the movement of any distinguishable shape or pattern in the images. The software output is a velocity vector contour-plot for the whole flowfield. If used for BOS application, the time gap between successive image is neglected and the software will produce displacement vector plot of the flowfield. This can easily be turned into density-gradient contour plot by relating the displacement of the dot-pattern with Gladstone-Dale equation. Absolute density at any point can then be calculated by solving Poisson equations for the whole flowfield[20]. The measured density can be used with known pressure to estimate the temperature (or vice versa) by using suitable equation of state [21]-[25].

Published By:

Blue Eyes Intelligence Engineering

\& Sciences Publication 
The cost of a commercial cross-correlation software is prohibitively high. This is due to limited number of supplier and the complexity of the cross-correlation numerical scheme. This has led to many researchers developing their own code. However, to develop one for our own case is beyond the scope of current lab exercise. Luckily, some limited-functionality but otherwise robust, PIV software can be found freely available on the internet. Some interesting ones include PIVlab[26], OpenPIV[27], PIVmat[28] and MatPIV[29]. The software were evaluated for ease of use and PIVlab was selected for the current project. We are more confident with this software since it has been used and validated by various research spanning many fields [30][36].

An old Digital Single-Lens Reflex camera was donated to this project by a colleague. The Nikon D90 camera can be purchased in second-hand market for about RM800, not including the lense. It was released in 2008, and was at one time considered the best of non-professional level Nikon DSLR. It can capture 12.3 megapixel of resolution and was the first to include $720 \mathrm{p}$ video recording capability. The lense used for this project was AF-S DX Nikkor with 18 $105 \mathrm{~mm}$ of focal length range and $\mathrm{f} 3.5$ of aperture. The price for the lense is around RM900 second hand. External lighting source was provided by Viltrox L116T Professional LED Video Light which can be bought online for RM 100. This LED light panel has dual colour temperature setting and 120 degrees light angle. The other important equipment is the tripod to hold the camera steady during experiments. A generic unbranded aluminium alloy tripod was bought from camera store for slightly less than RM200. The background needed for BOS was printed on a set of A4 papers and will be combined together on a plywood panel according to the total size needed. The pattern for the background was created using PIVmat application, an open source MATLAB toolbox created by [28]. Thus the total cost of the system is summarized in the Table 1 .

Table 1: Laboratory equipment and cost

\begin{tabular}{|c|c|}
\hline & Cost \\
\hline Nikon D90 Camera & RM 800 \\
\hline Nikkor $18-105 \mathrm{~mm} \mathrm{f3.5}$ & RM 900 \\
\hline Viltrox L116T Pro LED Video Light & RM 100 \\
\hline Aluminium Alloy Tripod & RM 200 \\
\hline PIVlab (BOS data processing software) & RM $\quad 0$ \\
\hline PIVmat (BOS pattern generator) & RM $\quad 0$ \\
\hline Total & RM 2000 \\
\hline
\end{tabular}

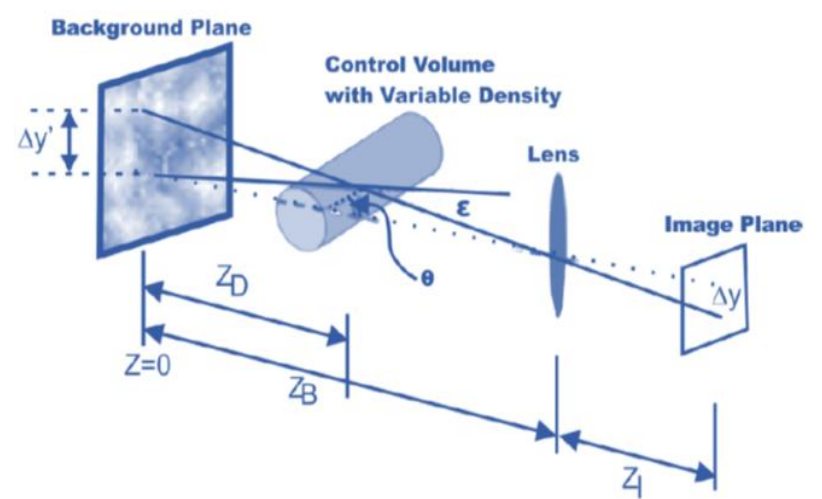

Fig. 2: Concept of background oriented Schlieren

\section{Developing the Laboratory Procedures}

We followed the example set by [37] in using the current postgraduate research projects as the basis in designing undergraduate laboratory exercises. This is to give the undergraduate students a taste of experimental research activities. Two master research project were selected; the first is a project of helicopter rotor tip enhancement and the other is a project of improving the combustion of a miniature combustor using vortex mixing.

The students were first briefed about the basic principal of BOS. The students were given the freedom to explore the software (PIVlab) and hardware capabilities on their own. Simple user manual for using PIVlab were given to the students. Typically, for many flow phenomena to be observed, the following procedures can be applied:

i. Download PIVlab with suitable version depending on MATLAB version already installed in Microsoft Windows based computer

ii. Open MATLAB and make sure installation folder of PIVLab is in the working directory

iii. Type PIVlab_gui in the command window and the graphical user interface such as in Fig. 3 will appear

iv. Click File $>$ New session

v. Click on Load images and choose a pair of images to be analyzed

vi. Click Analyses Settings $>$ Exclusions (ROI, Mask), and then drag a rectangular selection for region of interest (ROI)

vii. Click Analyses Settings $>$ PIV Settings, and pick either FFT for fast analysis or DCC for more precise analysis

viii. In the PIV settings, user needs to specify Interrogation Area window and its Step Size. The interrogation window will move across the whole ROI to scan any movement of patterns and its size is typically 64 pixel $\times 64$ pixel. The step size specify the amount of movement the interrogation window will make as it move across. The smaller the step size the more sensitive the movement detection will be. The suitable area and step size are very case dependant and must be decided by the students themselves

ix. Click Analysis>Analyze!>Analyze Current Frame, and the analysis will begin

x. Click Plot $>$ Derive Parameter, change the parameter to velocity magnitude and click the button Apply to Current Frame

Group meetings with designated lab instructor were held every week and the students were required to present their progress. They were evaluated based on their understanding of the objectives, procedure, safety precautions, and their expected results. Their engagement, whether they ask question or voicing out views and opinions, to the instructor and each other were also evaluated. All meeting sessions were recorded in a log-book for future review. The students were told to do their own readings of relevant literatures and must schedule their own working hours in four-weeks duration. 


\section{DEVELOPMENT OF LOW-COST THERMAL-FLUID UNDERGRADUATE LABORATORY EXERCISES COMPLYING WITH THE WASHINGTON ACCORD'S GRADUATE ATTRIBUTES}

At the end of a four-weeks period, they were required to present their final results in written form. The format for their report was typical of a scientific journal article. This is to give them the feel of contributing to the scientific community. They were judged based on their introduction, methodology, data analysis, discussion, conclusion and recommendations for future studies.

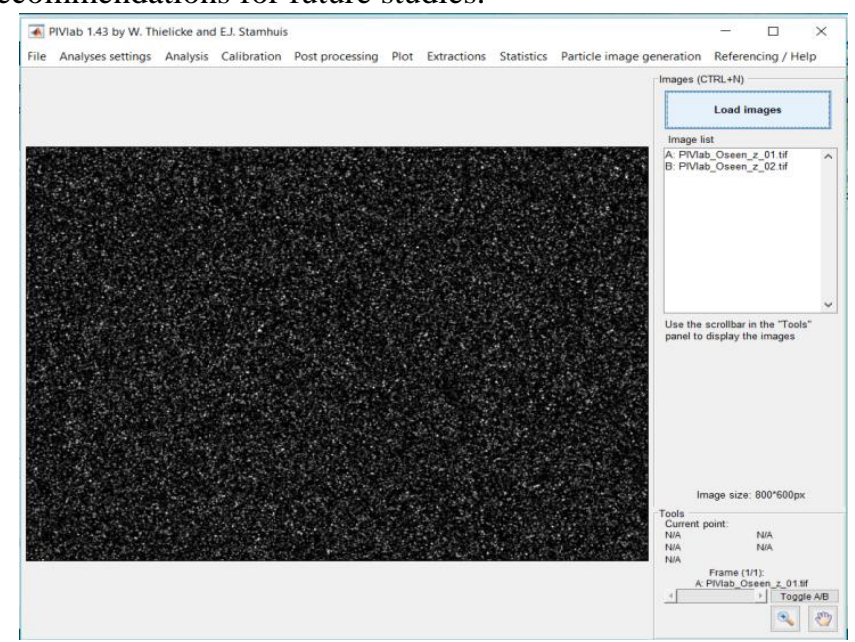

Fig. 3: Graphical User Interface (GUI) of PIVlab that will be used to measure the density gradient of flow phenomena in this experiments
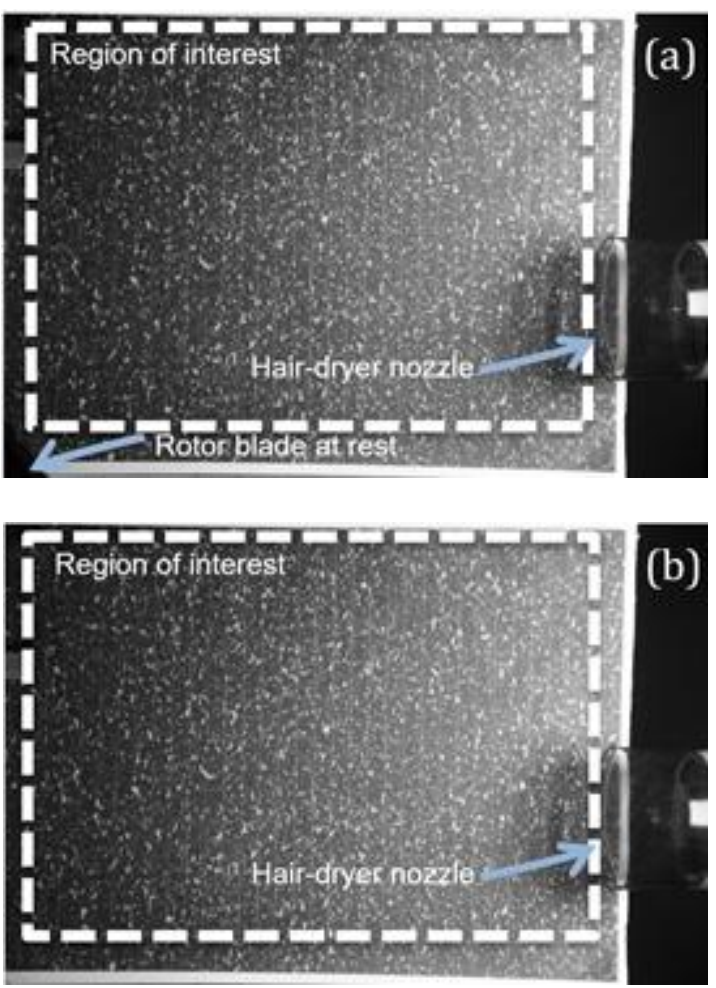

Fig. 4: Raw images of (a) wind-off, (b) wind-on

\section{RESULTS AND DISCUSSION}

\section{A. Investigation of Blade-Tip Vortex of an RC Helicopter}

It is well known that vortex will form at the tip of rotor blade and could cause "settling-with-power" or vortex-ring state where helicopter is flying in its own downwash [38]. This usually happens when the vortex from the blade tip grow exponentially and engulfing large segment of the rotor thus reducing overall lift. In this experiment, the students were required to visualize and comment on the vortex formation at the blade tip of a twin-rotor remote controlled helicopter. This experiment aims to replicate, in a small scale, the work done by the team in German Aerospace Center (DLR) where they visualize the blade tip vortex of a real helicopter in flight [39], [40]. The theory is that a vortex structure has different density in comparison to its surrounding air due to the condensation of air at its vortex core. This density gradient would allow it to be visualized using schlieren technique.

Since the model is small, the vortex emanating from the blade tip is very hard to visualize. The student found that increasing the number of patterns on the background would increase the overall sensitivity of BOS setup, however the rotor blade tip vortex still could not be detected. Based on further reading on fluid dynamics, the students found that the blade tip vortex, as a coherent flow structure, can interact with hot air from a hair-dryer thus further changing its density allowing it to be visualized using schlieren technique. The pre-processed images are shown in Fig. 4. In the wind-off (Fig. 4(a)), both the rotor and hair-dryer are off. The resultant density gradient plot is shown in Fig. 5 where we can observe high density gradient at the crosspath between the blade tip and the hot air exiting the hair-dryer. The hot air itself is barely visible but becomes highly apparent as it interacts with the flow-structure at the blade tip path. This flow-structure is most likely to be the blade tip vortex.

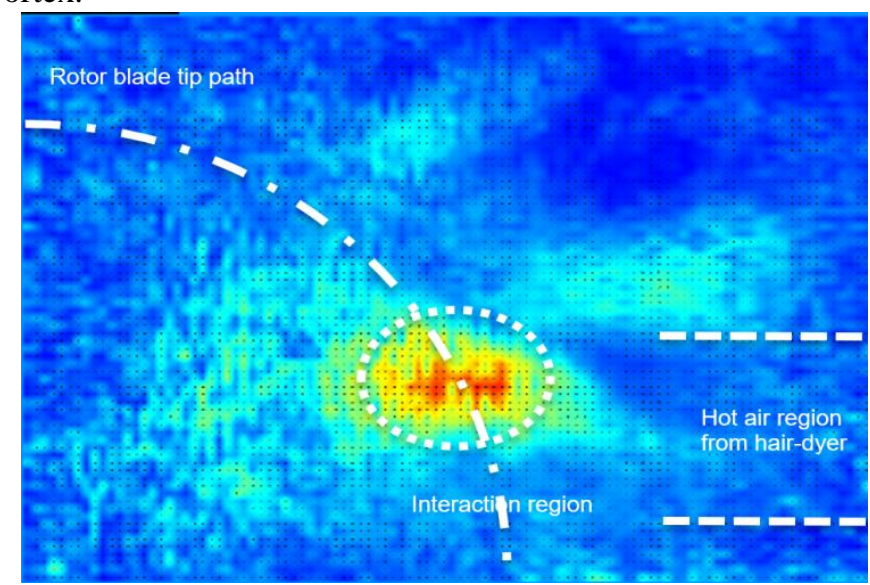

Fig. 5: Density gradient plot produced from analyzing the region of interest. Hot emanating from hair-dryer is visible only when it interacts with a flow-structure at the rotor tip

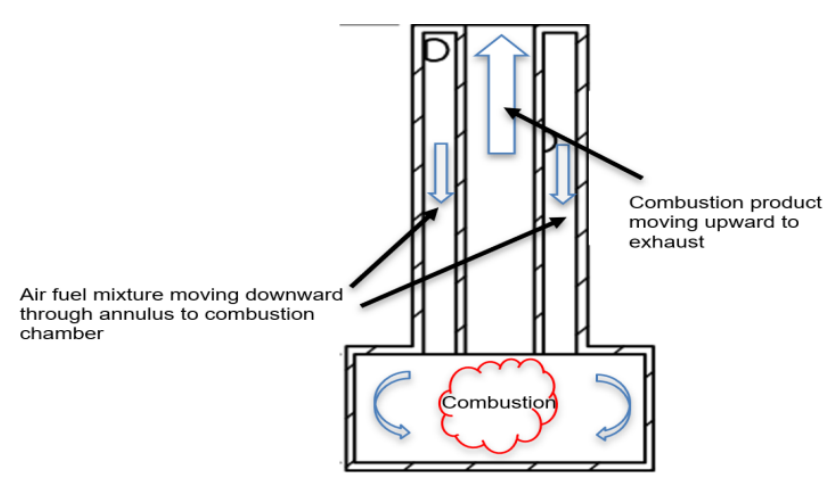

Fig. 6: Concept of reverse vortex trapped combustion

Blue Eyes Intelligence Engineering

\& Sciences Publication 


\section{B. Investigation of a Reverse-Trapped Vortex in a} Miniature Combustor

The concept of miniature combustor has been proposed as a portable power generator to be used with miniature devices in surveillance and communications [41], [42]. This type of combustor suffers from flame instability due to its large surface area to volume ratio in comparison to larger sized combustor [43]. Its small volume also discourage fuel/air mixing. To solve this problem, a miniature combustor has been designed in such a way it can trap the vortex from air and fuel injection inside the combustion chamber in order to confine the flame internally (see Fig. 6).
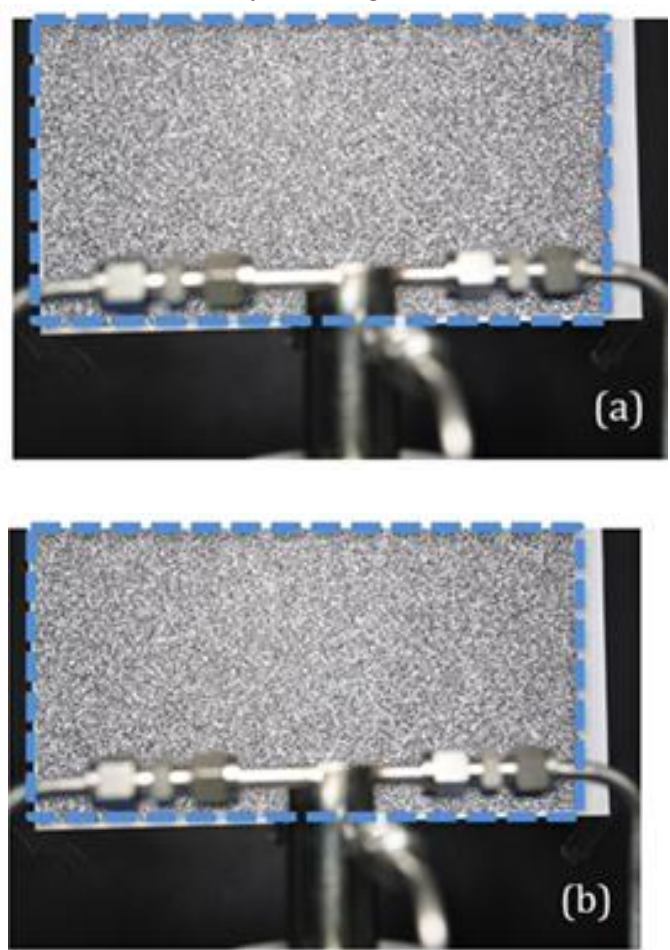

Fig. 7: Raw images of (a) wind-off, (b) wind-on

Fig. 7 shows the pre-processed images of combustion-off and combustion-on of the miniature combustor. It can be seen that no flame exited through exhaust. However, the postprocessed image in Fig. 8 is more interesting. It shows that the hot gas produced by the combustion expanded into the atmosphere in a non-symmetrical cone. Some coherent flowstructures can be observed suggesting the formation of first mode, single-helix jet flow as explained by Fiedler in his seminal paper [44]. This flow-structures are aided by the Rayleigh-Taylor instabilities due to the interaction between lower-density hot gas with the higher-density atmospheric air [45].

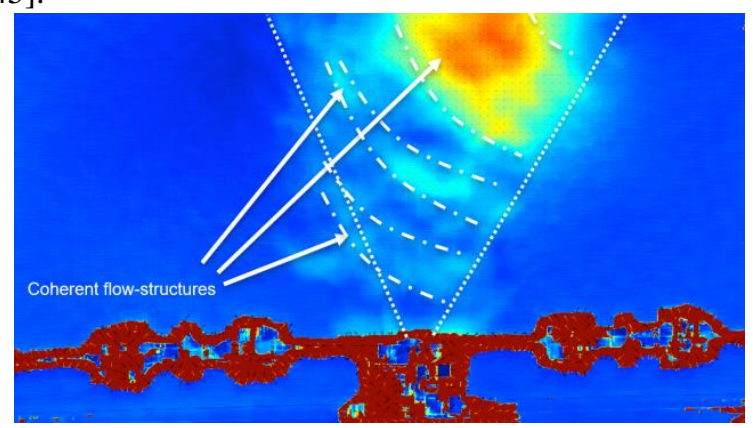

Fig. 8: Density gradient plot showing the exhaust gas produced by the miniature combustor

\section{Student Achievement on the Course Outcome}

The students were evaluated on their achievement of course outcome via their technical reports and logbooks that were filled for each lab sessions. They were also indirectly assessed via questionnaire on whether they themselves feel that they have achieved the course outcomes. Both assessments were averaged per number of student and compared in the Table 2 . The marks given by the instructor were very close to the level the students rate themselves. This shows that the students were able to work on complex engineering problems using modern tools that is currently on the cutting edge of research.

Table 2: Achievement of course outcome (CO) as assessed by the lab instructor and as the students felt they have achieved

\begin{tabular}{|l|l|l|}
\hline Course Outcomes from \\
\hline $\begin{array}{l}\text { Able to explain the theory } \\
\text { behind the experiment and } \\
\text { equipment }\end{array}$ & $\begin{array}{l}\text { Marks from } \\
\text { Instructor }\end{array}$ & $\begin{array}{l}\text { Marks } \\
\text { Questionnaire }\end{array}$ \\
\hline $\begin{array}{l}\text { Able to develop } \\
\text { appropriate experimental } \\
\text { objectives and } \\
\text { methodologies }\end{array}$ & $76 \%$ \\
\hline $\begin{array}{l}\text { Able to analyze, discuss, } \\
\text { conclude findings and } \\
\text { recommend future works }\end{array}$ & $71 \%$ & $76 \%$ \\
\hline $\begin{array}{l}\text { Able to conduct } \\
\text { experiments and prepare a } \\
\text { technical report as a team }\end{array}$ & $89 \%$ & $76 \%$ \\
\hline
\end{tabular}

\section{CONCLUSION}

An undergraduate laboratory teaching course, concentrating on thermal-fluid science, has been designed in order to satisfy the programmeoutcomes that is compliant with the Washington Accord. The laboratory exercise utilizes modern concept of BOS in order to measure and visualize the density gradient of many flow phenomena. The laboratory exercise was designed to be open-ended without a priorknown conclusion. The students did not expect what they will observe and were required to refer to scientific literatures on their own. The students were able to describe their observation and participate actively in a weekly discussions with their respective lab instructor, thus training them to become highly inquisitive researchers in the future.

\section{REFERENCES}

1 L. Brodie, F. Bullen, and L. Jolly, "Effective evaluation strategies to meet global accreditation requirements," Frontiers in Education Conference, 2011, pp. S1B-1S1B-6.

2 Board of Engineers Malaysia (BEM), Engineering programme accreditation manual 2012. Kuala Lumpur: BEM.

3 Board of Engineers Malaysia (BEM), Engineering programme accreditation manual 2017. Kuala Lumpur: BEM. 


\section{DEVELOPMENT OF LOW-COST THERMAL-FLUID UNDERGRADUATE LABORATORY EXERCISES COMPLYING WITH THE WASHINGTON ACCORD'S GRADUATE ATTRIBUTES}

4 L. M. S. Khoo, D. Maor, and R. Schibeci, "The engineering eportfolio: Enhancing communication, critical thinking and problem solving and team work skills?," World Academy of Science, Engineering and Technology, 77, 2011, pp. 1027-1032.

5 N. A. Kadir and M. Arsat, Kemahiran generik dalam faktor pemilihan jurutera mengikut perspektif industri pembinaan. 2009, Available: Http://www.fp.utm.my/epusatsumber/pdffail/ptkghdfwp2 /p_2009_8778_cd021bb92c44414bae6039891948e717.p df.

6 Z. Hanapi, M. S. Nordin, and R. C. Rus, "Unemployment problem among graduates of technical field: Competencies of the graduates and quality of the education," Sains Humanika, 2(2), 2014, pp. 53-57.

7 Y. Cengel, R. Turner, and J. Cimbala, Fundamentals of Thermal-Fluid Sciences. New York: McGraw Hill Education, 2017.

8 A. C. Idris, Characterization of high speed inlets using global measurement techniques. $\mathrm{PhD}$ thesis, England: University of Manchester, 2014.

9 B. P. Ring and E. Lemley, "Design and implementation of a low cost particle image velocimetry system for undergraduate research and education," ASEE Annual Conference and Exposition, 2014, pp. 1-11.

10 B. P. Ring, D. K. Atkinson, A. W. Henderson, and E. Lemley, "Development of a low cost particle image velocimetry system for fluids engineering research and education," ASME 2013 Fluids Engineering Division Summer Meeting, 2013, pp. 1-5

11 U. Rivera-Ortega, N. Martinez-Sisniega, and U. Alcantara-Mendoza, "Low-cost educational resource using optical fibers to send 4-bit images in grayscale," Computer Applications in Engineering Education, 26(3), 2018, pp. 711-717.

12 I. Uyanik and B. Catalbas, "A low-cost feedback control systems laboratory setup via Arduino-Simulink interface," Computer Applications in Engineering Education, 26(3), 2018, pp. 718-726.

13 H. Mostefaoui, A. Benachenhou, and A. A. Benattia, "Design of a low cost remote electronic laboratory suitable for low bandwidth connection," Computer Applications in Engineering Education, 25(3), 2017, pp. 480-488.

14 J. García and J. Entrialgo, "Using computer virtualization and software tools to implement a low cost laboratory for the teaching of storage area networks," Computer Applications in Engineering Education, 23(5), 2015, pp. 715-723.

15 F. Botana, M. A. Abánades, and J. Escribano, "Using a free open source software to teach mathematics," Computer Applications in Engineering Education, 22(4), 2014, pp. 728-735.

16 T. Inanc and H. Dinh, "A low-cost autonomous mobile robotics experiment: Control, vision, sonar, and Handy Board," Computer Applications in Engineering Education, 20(2), 2012, pp. 203-213.

17 O. Galland, H. S. Bertelsen, F. Guldstrand, L. Girod, R. F. Johannessen, F. Bjugger, S. Burchardt, and K. Mair, "Application of open-source photogrammetric software MicMac for monitoring surface deformation in laboratory models," Journal of Geophysical Research: Solid Earth, 121(4), 2016, pp. 2852-2872

18 D. Mohapatra, N. Kashyap, A. P. Biswal, and S. Padhee, "Design of measurement and data acquisition laboratory for instrumentation engineering course," 3rd International Conference on Computational Intelligence Communication Technology, 2017, pp. 1-6.

19 G. S. Settles, Schlieren and Shadowgraph Techniques: Visualizing Phenomena in Transparent Media. Berlin: Springer Science and Business Media, 2001.
20 M. Raffel, "Background-oriented schlieren (BOS) techniques," Experiments in Fluids, 56(3), 2015, pp. 60.

21 S. Tokgoz, R. Geisler, L. J. A. van Bokhoven, and B. Wieneke, "Temperature and velocity measurements in a fluid layer using background-oriented schlieren and PIV methods," Measurement Science and Technology, 23(11), 2012, pp. 1-10.

22 A. Blanco, B. Barrientos, and C. Mares, "Performance comparison of background-oriented schlieren and fringe deflection in temperature measurement: Part I. Numerical evaluation," Optical Engineering, 55(5), 2016, pp. 1-9.

23 N. A. Vinnichenko, A. V. Uvarov, and Y. Y. Plaksina, "Combined study of heat exchange near the liquid-gas interface by means of background oriented schlieren and infrared thermal imaging," Experimental Thermal and Fluid Science, 59, 2014, pp. 238-245.

24 M. J. Hargather and G. S. Settles, "A comparison of three quantitative schlieren techniques," Optics and Lasers in Engineering, 50(1), 2012, pp. 8-17.

25 G. Tanda, M. Fossa, and M. Misale, "Heat transfer measurements in water using a schlieren technique," International Journal of Heat and Mass Transfer, 71, 2014, pp. 451-458

26 W. Thielicke and E. Stamhuis, "PIVlab - Towards userfriendly, affordable and accurate digital particle image velocimetry in MATLAB," Journal of Open Research Software, 2(1), 2014, pp. 1-10.

27 Z. J. Taylor, R. Gurka, G. A. Kopp, and A. Liberzon, "Long-duration time-resolved PIV to study unsteady aerodynamics," IEEE Transactions on Instrumentation and Measurement, 59(12), 2010, pp. 3262-3269.

28 F. Moisy, M. Rabaud, and E. Pinsolle, "Measurement by digital image correlation of the topography of a liquid interface," 13th International Symposium on Flow Visualization, and 12th French Congress on Visualization in Fluid Mechanics, Paper, no. 326, 2008, pp. 8.

29 J. K. Sveen and E. A. Cowen, "Quantitative imaging techniques and their application to wavy flows," PIV and Water Waves, 2004, pp. 1-49.

30 A. C. Idris, M. R. Saad, K. H. Lo, and K. Kontis, "Background-oriented schlieren (BOS) for scramjet inletisolator investigation," IOP Conference Series: Materials Science and Engineering, 370(1), 2018, pp. 1-8.

31 K. T. Wu, J. B. Hishamunda, D. T. N. Chen, S. J. DeCamp, Y. W. Chang, A. Fernández-Nieves, S. Fraden, and Z. Dogic, "Transition from turbulent to coherent flows in confined three-dimensional active fluids," Science, 355(6331), 2017, pp. 1-9.

32 D. J. Asselin and C. H. K. Williamson, "Influence of a wall on the three-dimensional dynamics of a vortex pair," Journal of Fluid Mechanics, 817, 2017, pp. 339-373

33 S. Roman, C. Soulaine, M. A. AlSaud, A. Kovscek, and H. Tchelepi, "Particle velocimetry analysis of immiscible two-phase flow in micromodels," Advances in Water Resources, 95, 2016, pp. 199-211.

34 O. Ghaffari, S. A. Solovitz, M. Ikhlaq, and M. Arik, "An investigation into flow and heat transfer of an ultrasonic micro-blower device for electronics cooling applications," Applied Thermal Engineering, 106, 2016 pp. 881-889.

35 Y. Bashirzadeh, V. Maruthamuthu, and S. Qian, "Electrokinetic phenomena in pencil lead-based microfluidics," Micromachines, 7(12), 2016, pp. 1-13. 
36 M. Mohammadzadeh, S. R. Gonzalez-Avila, K. Liu, Q. J. Wang, and C.-D. Ohl, "Synthetic jet generation by highfrequency cavitation," Journal of Fluid Mechanics, 823, 2017, pp. 1-12.

37 N. M. Komerath, "Experimental curriculum in diagnostics and control of unsteady flows," Journal of Engineering Education, 85(3), 1996, pp. 263-268.

38 A. Filippone, Flight Performance of Fixed and Rotary Wing Aircraft. Massachusetts: Butterworth-Heinemann, 2006.

39 H. Richard and M. Raffel, "Principle and applications of the background oriented schlieren (BOS) method," Measurement Science and Technology, 12(9), 2001, pp. 1576-1585.

40 M. Raffel, C. Tung, H. Richard, Y. Yu, and G. E. A. Meier, "Background oriented stereoscopic schlieren (BOSS) for full scale helicopter vortex characterization," 9th International Symposium on Flow Visualization, 2000, pp. 23-24.

41 S. K. Chou, W. M. Yang, K. J. Chua, J. Li, and K. L. Zhang, "Development of micro power generators - A review," Applied Energy, 88(1), 2011, pp. 1-16.

42 V. Shirsat and A. K. Gupta, "A review of progress in heat recirculating meso-scale combustors," Applied Energy, 88(12), 2011, pp. 4294-4309.

$43 \mathrm{Y}$. Ju and $\mathrm{K}$. Maruta, "Microscale combustion: Technology development and fundamental research," Progress in Energy and Combustion Science, 37(6), 2011, pp. 669-715.

44 H. E. Fiedler, "Coherent structures in turbulent flows," Progress in Aerospace Sciences, 25(3), 1988, pp. 231269.

45 H. J. Kull, "Theory of the Rayleigh-Taylor instability," Physics Reports, 206(5), 1991, pp. 197-325.

\section{AUTHORS PROFILE}

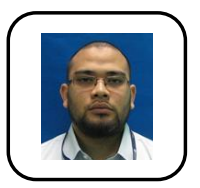

Ts. Dr. AzamCheIdris CEng completed his MEng and $\mathrm{PhD}$ both from the University of Manchester in 2009 and 2013, respectively. His $\mathrm{PhD}$ study was on the development of novel thermal-fluid diagnostic methods capable of characterizing the scramjet intake. $\mathrm{He}$ is currently the Coordinator for Head of Labs, overseeing all 16 laboratories in the mechanical engineering department in UniversitiPertahananNasional Malaysia (UPNM).

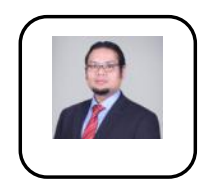

Dr. MohdRashdanSaad completed his MEng and PhD both from the University of Manchester in 2009 and 2013, respectively. His PhD study was on Micro-Vortex Generators (MVG) in controlling shock boundary layer interactions (SBLI) in hypersonic flow. The study involves the use of novel wind tunnel flow diagnostic to understand the flow physics of MVG and its effectiveness in controlling SBLI. Upon finishing his studies, he returned to Malaysia and was being appointed as a lecturer at the Faculty of Engineering, UniversitiPertahananNasional Malaysia (UPNM).

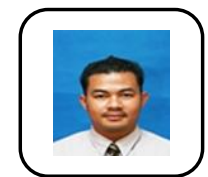

SuriyadiSojipto is a lecturer with the Faculty of Engineering, UniversitiPertahananNasional Malaysia, Kuala Lumpur. His research interests are steel and reinforced concrete structures subjected to extreme loading explosion and fire.

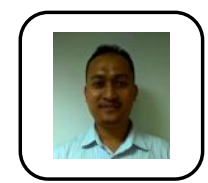

Ir. Dr. MohdRosdzimin Abdul Rahman is a Senio Lecturer in the Department of Mechanical Engineering, UniversitiPertahananNasional Malaysia. He also served as the Research Fellow for Centre for Defence Research and Technology (CODRAT). He works in a wide range of thermal and reactive fluid dynamics numerically and experimentally, including combustion/fire dynamics, thermal management, nanofluid as well as meso-scale combustor. He has also produced more than 60 publications to date in internationally refereed journals, conference proceedings and book chapters. 